\title{
Efeitos do Treinamento de Resistência de Força com Alto Número de Repetições no Consumo Máximo de Oxigênio e Limiar Ventilatório de Mulheres
}

\author{
Effects of Strength Resistance Training With High Number of Repetitions \\ on Maximal Oxygen Uptake and Ventilatory Threshold in Women
}

Thiago Mattos Frota de Souza Marcelo de Castro Cesar ${ }^{1}$ João Paulo Borin Pamela Roberta Gomes Gonelli Ricardo Adamoli Simões Maria Imaculada de Lima Montebelo²

1. Núcleo de Performance Humana - Curso de Educação Física

- Faculdade de Ciências da Saúde - Universidade Metodista de Piracicaba (Unimep) - São Paulo - Brasil.

2. Faculdade de Ciências Exatas e da Natureza - Universidade Metodista de Piracicaba (Unimep) - São Paulo - Brasil.

Endereço para correspondência: Thiago Mattos Frota de Souza, Rua Padre José Conceição Meirelles, 30 - 13418-390 - Piracicaba - São Paulo, Brasil. Tel.: (19) 3425-1641. Celular: (19) 9669-1049.

E-mail: thiago_mfs@hotmail.com

Submetido em 19/10/2007

Versão final recebida em 05/05/2008 Aceito em 05/07/2008

\section{RESUMO}

Os efeitos do treinamento com pesos na aptidão cardiorrespiratória de mulheres não estão amplamente definidos. O estudo teve como objetivo investigar os efeitos do treinamento de resistência de força com alto número de repetições no consumo máximo de oxigênio e limiar ventilatório em mulheres jovens. Participaram deste estudo 20 mulheres, com idade de 21,2 +2,7 anos, agrupadas em: I - grupo de treinamento de resistência de força com alto número de repetições (GT, $n=10)$ e II - controle (GC, $\mathrm{n}=10$ ). Todas as voluntárias foram submetidas ao seguinte protocolo: antropometria, teste cardiopulmonar em esteira rolante e testes de 1RM nos exercícios: leg-press 450, cadeira extensora, mesa flexora, supino reto, puxador costas, desenvolvimento com a barra, rosca direta e tríceps com a barra. Os testes foram realizados antes e após 12 semanas. No período proposto, os grupos realizaram: I - GT: exercícios resistidos, os mesmos dos testes de 1RM, com três séries de 25 repetições, com aproximadamente 30\% de 1RM; II - GC: não realizou nenhum treinamento físico. Após 12 semanas, o GC não apresentou alterações nas variáveis estudadas $(p>0,05)$. O GT apresentou aumento significante da massa magra $(p<0,05)$ e diminuição do percentual de gordura $(p<0,01)$ e da gordura corporal $(p<0,01)$. Houve aumento dos testes de 1RM em todos os exercícios $(p<0,01)$ e aumento do consumo máximo de oxigênio $(p<0,05)$, mas não houve alteração no limiar ventilatório $(p>0,05)$. Conclui-se que o treinamento de resistência de força com alto número de repetições proporcionou melhora da potência aeróbia das voluntárias, evidenciado pelo aumento do consumo máximo de oxigênio, embora não tenha modificado o limiar ventilatório.

Palavras-chave: força muscular, avaliação, variáveis cardiorrespiratórias, exercício físico.

\section{ABSTRACT}

The effects of strength training on women's cardiorespiratory capacity have not been widely established. The purpose of this study was to investigate the effects of resistance strength training with high number of repetitions on maximum oxygen uptake and ventilatory threshold in young women during 12 weeks. Twenty women, aged $21.2+2.7$ years, were included in the study and assigned to two groups: I - resistance strength training with high number of repetitions group ( $T, \mathrm{n}=10$ ), and || - control group $(C G, n=10)$. All volunteers were submitted to the following protocol: anthropometry, cardiopulmonary testing on treadmill and 1 RM tests in the following exercises: leg-press 450, seated leg extension, hamstring curl, bench press, lat pull-down, military press, standing barbell curls and lying barbell extension. The tests were performed before and after 12 weeks of training. Along the proposed period, the groups performed: I -TG: resistance exercises, the same of 1 RM tests, with three series of 25 repetitions, almost 30\% of 1 RM; II - CG: no physical training whatsoever. CG did not show changes in the studied variables $(p<0.05)$. TG showed significant increase of lean mass $(p<0.05)$ and decrease of fat percentage $(p<0.01)$ and body fat $(p<0.01)$. There was increase in 1 RM tests in all exercises $(p<0.01)$ and increase of maximum oxygen uptake $(p<0.05)$; however, there was not change in the ventilatory threshold $(p>0.05)$ after 12 weeks. It is concluded that the resistance strength training with high number of repetitions improved the aerobic power of volunteers, evidenced by the increase of maximum oxygen uptake. However, the ventilatory threshold did not change.

Keywords: muscle strength, assessment, cardiorespiratory variables, physical exercise. 


\section{INTRODUÇÃO}

O treinamento de força, realizado com pesos, é utilizado com vários objetivos, como melhorar o rendimento em esportes, o condicionamento físico, a estética e para a promoção da saúde ${ }^{(1-3)}$, possuindo efeitos benéficos no aumento da força muscular, potência e resistência anaeróbia ${ }^{(3)}$. A força muscular pode ser classificada em força máxima, força rápida e resistência de força, que é a capacidade de resistir à fadiga em condições de desempenho prolongado da força ${ }^{(4)}$.

O treinamento de resistência de força é o mais utilizado com fins de condicionamento físico e promoção de saúde em academias. É classificado como treinamento de resistência de força com alto número de repetições aquele a partir de 15 repetições e curtos intervalos de repouso, de 30 a 60 segundos de descanso entre as séries ${ }^{(3-5)}$.

Enquanto as respostas do treinamento aeróbio no aumento da aptidão cardiorrespiratória são positivas ${ }^{(6,7)}$, os efeitos do treinamento de força no consumo máximo de oxigênio $\left(\mathrm{VO}_{2 \max }\right)$ e limiar anaeróbio (LA), que são os dois principais índices da aptidão cardiorrespiratória ${ }^{(8,9)}$, ainda necessitam de maior investigação. Alguns estudos demonstraram que o treinamento de força não proporciona aumento no $\mathrm{VO}_{2 \max }{ }^{(10-13)}$, enquanto outros evidenciaram o contrário ${ }^{(14-16)}$, ou que ainda não encontraram aumento no LA, conforme evidenciaram Hoff et al. ${ }^{(11)}$ ao submeter atletas de esqui do sexo feminino ao treinamento de força. Apesar de os treinamentos aeróbios e de força levarem a adaptações musculares distintas, de acordo com os princípios do treinamento ${ }^{(17)}$, o treinamento de força com alto número de repetições e curtos intervalos de descanso apresenta importante contribuição do metabolismo aeróbio ${ }^{(3-5)}$. Ou seja, o treinamento aeróbio diminui a atividade das enzimas glicolíticas, mas aumenta os estoques intramusculares de substrato energético, enzimas oxidativas, capilarização e a densidade mitocondrial, enquanto que o treinamento de força reduz a densidade mitocondrial e tem pequeno efeito na capilarização, enzimas oxidativas e estoques de substratos intramusculares, exceto glicogênio muscular ${ }^{(17)}$. Já o treinamento de resistência de força com alto número de repetições e curtos intervalos de descanso, características de resistência muscular localizada, estimula o aumento do número de mitocôndrias, capilarização, transições de tipos de fibras e capacidade-tampão dentro do músculo esquelético(3).

Embora as respostas ao treinamento de homens e mulheres possam ser semelhantes, a magnitude das adaptações femininas difere da das masculinas ${ }^{(18)}$. De acordo com Bishop et al. ${ }^{(10)}$, as respostas da testosterona ao treinamento com pesos, diferentes em homens em relação às mulheres, poderiam explicar as diferentes adaptações ao treinamento entre os gêneros.

Sendo assim, e devido à grande participação de mulheres envolvidas em programas de treinamento com pesos, o objetivo do presente estudo foi avaliar os efeitos do treinamento de resistência de força com alto número de repetições no consumo máximo de oxigênio e no limiar ventilatório de mulheres jovens.

\section{MÉTODOS}

\section{Sujeitos}

Participaram deste estudo 20 mulheres, idade de 21,2 $\pm 2,7$ anos, saudáveis, não treinadas e não fazendo uso de medicamentos. Foram consideradas não treinadas as voluntárias que não participavam de um programa de treinamento físico pelo menos três meses antes, além de não estarem envolvidas em nenhum tipo de exercícios regulares durante a realização do estudo(19). As voluntárias foram subdivididas em dois grupos:
- Grupo I - Experimental (GE): 10 mulheres, que foram submetidas a um programa de treinamento de resistência de força com alto número de repetições.

- Grupo II - Controle (GC): 10 mulheres, que não foram submetidas a nenhum treinamento físico.

Após a explicação do estudo, as voluntárias assinaram um termo de consentimento livre e esclarecido. O presente estudo foi aprovado pelo Comitê de Ética em Pesquisa da Universidade Metodista de Piracicaba (protocolo no. 83/03).

\section{Avaliação antropométrica}

A avaliação antropométrica foi realizada antes e após 12 semanas do estudo. As medidas foram coletadas respeitando-se a seguinte ordem: estatura (utilizando o estadiômetro Alturaexata"), massa corporal (utilizando a balança mecânica Welmy") e dobras cutâneas subescapular, supra-ilíaca e coxa (utilizando o plicômetro Lange ${ }^{\circ}$ ). Para a determinação do percentual de gordura, gordura corporal absoluta e massa magra utilizou-se o protocolo proposto por Guedes \& Guedes ${ }^{(20)}$.

\section{Teste cardiopulmonar}

Os dois grupos foram submetidos ao teste cardiopulmonar antes e após 12 semanas, em laboratório climatizado, com temperatura mantida entre $20^{\circ}$ e $24^{\circ} \mathrm{C}$, em uma esteira rolante computadorizada (Inbrasport ATL ${ }^{\circ}$, com protocolo contínuo, de carga crescente, com carga inicial de $4,0 \mathrm{~km} / \mathrm{h}$ (durante dois minutos), com incrementos de $1,0 \mathrm{~km} / \mathrm{h}$ a cada minuto, até $10,0 \mathrm{~km} / \mathrm{h}$, a seguir, incrementos de $2,5 \%$ de inclinação/minuto, até a exaustão(7).

A medida do consumo de oxigênio, gás carbônico e da ventilação pulmonar foi realizada de forma direta, a cada 20 segundos, por analisador de gases metabólicos (VO2000 - Aerosport Medical Graphics'). Foram determinados o consumo máximo de oxigênio $\left(\mathrm{VO}_{2 \max }\right)$ e o limiar ventilatório (LV), expressos em mililitros por quilograma por minuto $(\mathrm{ml} / \mathrm{kg} / \mathrm{min})$.

$\mathrm{OVO}_{2 \max }$ foi determinado pelo menos por três dos seguintes critérios: quando o consumo de oxigênio atingia um platô (variação $<2 \mathrm{ml} /$ $\mathrm{kg} / \mathrm{min}$ ) mesmo com o aumento da intensidade do esforço, a razão das trocas respiratórias $(R) \geq 1,10$, pequena variação da freqüência cardíaca em esforço máximo, ou seja, variação de \pm 5 bpm da freqüência cardíaca máxima prevista para a idade, e quando as voluntárias atingiam a fadiga máxima que as impedia, voluntariamente, de continuar o esforço, ou seja, percepção subjetiva de esforço (escala de Borg) > 17(8,21).

O limiar anaeróbio foi determinado por método ventilatório (limiar ventilatório), pelos seguintes $\operatorname{critérios}^{(9)}$ : hiperventilação pulmonar, aumento sistemático do equivalente ventilatório para o oxigênio, aumento abrupto da razão de trocas gasosas.

A freqüência cardíaca durante o teste foi determinada pelos intervalos $\mathrm{R}-\mathrm{R}$ (derivações $\mathrm{MC}_{5}, \mathrm{AVF} \mathrm{eV}_{2}$ ) do eletrocardiograma (eletrocardiógrafo Dixtal EP3 $3^{\circ}$ ) e expressa em batimentos por minuto (bpm).

\section{Testes de força máxima}

Para a determinação dos efeitos do treinamento de resistência de força com alto número de repetições na força muscular, foram realizados os testes de uma repetição máxima (1RM), como descrito por Brown \& Weir (22), referindo-se à quantidade máxima de peso levantada em um movimento correto de um exercício padronizado. A cada repetição realizada, foi acrescentada uma carga extra ao dispositivo do exercício, até alcançar o limite máximo; no caso de fadiga, um peso intermediário entre o último levantamento de sucesso e o de fadiga era tentado, determinando a 1RM no nível desejado de precisão. 
Foram realizados os testes nos seguintes exercícios: leg-press 45, cadeira extensora, mesa flexora, supino reto, puxador costas, desenvolvimento com a barra, rosca direta e tríceps com a barra.

\section{Treinamento de resistência de força}

As 10 mulheres do GE foram submetidas a um treinamento de resistência de força com alto número de repetições, consistindo em três séries de 25 repetições com cerca de 30\% de 1RM, nos mesmos exercícios utilizados nos testes de 1RM. O programa de treinamento ocorreu três vezes por semana, com duração de uma hora (cada sessão), durante 12 semanas.

Antes de cada sessão de treinamento, as voluntárias realizavam aquecimento e alongamento com exercícios balísticos e 10 repetições a cerca de $10 \%$ da carga de treinamento nos exercícios supino reto, puxador costas e leg-press $45^{\circ}$. Os exercícios foram realizados com intervalos de 60 segundos entre as séries. A determinação das cargas de treinamento ocorreu de acordo com o critério de repetições máximas $(\mathrm{RM})^{(3,5)}$; as cargas foram determinadas no decorrer das três primeiras sessões, por meio de treinamento individualizado da carga possível para a realização das RM.

\section{Plano analítico}

Foi realizada a análise exploratória dos dados e foram verificadas as pressuposições para o uso dos métodos estatísticos paramétricos. Para avaliar a pressuposição de normalidade, utilizou-se o teste de ShapiroWilk. Para avaliar a igualdade das variâncias entre os grupos, ou seja, a homocidasticidade, utilizou-se o teste de Levene ${ }^{(23)}$.

As análises foram processadas por meio do programa SPSS, os resultados descritos em média e desvio-padrão, considerando-se o nível de significância de 5\% ( $p<0,05)$.

Para a comparação entre os grupos foi utilizado o teste $t$ de Student e o de Mann-Whitney para amostras independentes. A comparação das variáveis observadas antes e após o treinamento para cada grupo foi realizada utilizando-se o teste $t$ de Student e o teste de Wilcoxon para amostras pareadas.

\section{RESULTADOS}

No início do estudo nenhuma das variáveis apresentou diferença significante entre os grupos GE e GC.

Após o treinamento, na avaliação antropométrica do GE houve significante aumento da massa magra e diminuição do percentual de gordura e da gordura corporal, não ocorrendo diferenças significantes da massa corporal. No grupo controle não houve diferenças significantes das medidas entre o início e o final (tabela 1).

Tabela 1. Valores da antropometria dos grupos experimental (GE) e controle (GC)

\begin{tabular}{l|c|c|c|c}
\hline \multirow{2}{*}{ Variáveis } & \multicolumn{2}{|c|}{ GE $(\mathbf{n}=10)$} & \multicolumn{2}{c}{ GC (n=10) } \\
\cline { 2 - 5 } & Pré-treinamento & Pós-treinamento & Inicial & Final \\
\hline MC (kg) & $59,2 \pm 6,4$ & $59,2 \pm 6,9$ & $58,1 \pm 8,8$ & $57,6 \pm 8,5$ \\
\hline$\%$ Gord. (\%) & $24,0 \pm 2,8$ & $22,0 \pm 3,6^{* *}$ & $24,8 \pm 3,2$ & $24,6 \pm 3,1$ \\
\hline GC (kg) & $14,3 \pm 2,7$ & $13,1 \pm 3,0^{* *}$ & $14,6 \pm 3,9$ & $14,4 \pm 3,7$ \\
\hline MM (kg) & $44,9 \pm 4,3$ & $46,1 \pm 4,9 *$ & $43,5 \pm 5,1$ & $43,2 \pm 5,1$ \\
\hline
\end{tabular}

Valores são média \pm desvio-padrão. MC = Massa corporal; IMC = Indice de massa corporal; \%Gord. = Percentual de gordura; GC = Gordura corporal; MM = Massa magra. Para a comparação das variáveis antes e após 12 semanas foi utilizado o teste $t$ de Student para amostras pareadas para: MC (GE e GC), \%Gord. (GC), GC (GE e GC) e MM (GE e GC); para o \%Gord. (GE), foi utilizado o teste de Wilcoxon. * Indica diferença significante antes e depois do treinamento $(p<0,05)$. ** Indica diferença significante antes e depois do treinamento $(p<0,01)$.
As voluntárias do GE iniciaram o treinamento em média a 31,3\% de 1 RM e finalizaram a 34,5\% de 1RM. Nos testes de 1RM, as voluntárias do GE apresentaram aumento significante em todos os exercícios, não ocorrendo diferenças significantes no GC (tabela 2).

Tabela 2. Valores dos testes de 1RM dos grupos experimental (GE) e controle (GC)

\begin{tabular}{l|c|c|c|c}
\hline \multirow{2}{*}{$\begin{array}{c}\text { Variáveis } \\
\text { (kg) }\end{array}$} & \multicolumn{2}{|c|}{ GE (n=10) } & \multicolumn{2}{c}{ GC (n=10) } \\
\cline { 2 - 5 } & Pré-treinamento & Pós-treinamento & Inicial & Final \\
\hline Supino & $29,2 \pm 5,1$ & $34,0 \pm 5,1^{* *}$ & $27,9 \pm 4,7$ & $27,7 \pm 4,3$ \\
\hline Puxador & $24,6 \pm 5,8$ & $30,9 \pm 5,8^{* *}$ & $26,4 \pm 4,2$ & $25,5 \pm 3,9$ \\
\hline Desenv. & $22,9 \pm 3,5$ & $26,6 \pm 3,2^{* *}$ & $22,6 \pm 4,5$ & $23,0 \pm 3,7$ \\
\hline Rosca & $18,0 \pm 2,5$ & $20,4 \pm 3,4^{* *}$ & $16,0 \pm 3,5$ & $15,8 \pm 2,1$ \\
\hline Tríceps & $12,6 \pm 2,1$ & $16,2 \pm 3,1 * *$ & $14,0 \pm 2,2$ & $13,8 \pm 2,7$ \\
\hline LP 450 & $130,5 \pm 27,7$ & $167,6 \pm 25,9 * *$ & $127,4 \pm 37,7$ & $127,3 \pm 36,5$ \\
\hline Extens. & $25,8 \pm 4,3$ & $32,4 \pm 7,0 * *$ & $28,9 \pm 7,5$ & $27,7 \pm 6,7$ \\
\hline Flexão & $31,8 \pm 8,1$ & $37,8 \pm 8,9 * *$ & $30,6 \pm 7,6$ & $30,6 \pm 7,2$ \\
\hline
\end{tabular}

Valores são média \pm desvio-padrão. Supino = Supino reto com a barra; Puxador = Puxador costas; Desenv.$=$ Desenvolvimento com a barra; Rosca $=$ Rosca direta com a barra; Tríceps $=$ Tríceps com a barra; LP 45 = Leg-press 45; Extens. = Extensão de joelhos na cadeira extensora; Flexão = Flexão de joelhos na mesa flexora. Para a comparação das variáveis antes e após 12 semanas foi utilizado o teste $t$ de Student para amostras pareadas. * Indica diferença significante antes e depois do treinamento $(p<0,05)$. ${ }^{* *}$ Indica diferença significante antes e depois do treinamento $(p<0,01)$.

No teste cardiopulmonar, o GE apresentou diferenças significantes no $\mathrm{VO}_{2 \max }$ e pulso máximo de oxigênio após o treinamento, não alterando significantemente o LV. O GC não apresentou diferenças significantes entre as medidas inicial e final (tabela 3).

Tabela 3. Variáveis cardiopulmonares dos grupos experimental (GE) e controle (GC)

\begin{tabular}{|c|c|c|c|c|}
\hline \multirow{2}{*}{ Variáveis } & \multicolumn{2}{|c|}{$G E(n=10)$} & \multicolumn{2}{|c|}{$G C(n=10)$} \\
\hline & Pré-treinamento & Pós-treinamento & Inicial & Final \\
\hline $\mathrm{VO}_{2 \max }(\mathrm{ml} / \mathrm{kg} / \mathrm{min})$ & $35,3 \pm 6,2$ & $37,0 \pm 6,4^{*}$ & $34,2 \pm 3,5$ & $34,3 \pm 3,8$ \\
\hline FCM (bpm) & $196,4 \pm 4,5$ & $197,5 \pm 4,2$ & $196,2 \pm 4,0$ & $195,7 \pm 3,9$ \\
\hline $\mathrm{VO}_{2 \max }(\mathrm{l} / \mathrm{min})$ & $2,1 \pm 0,4$ & $2,2 \pm 0,4^{* *}$ & $2,0 \pm 0,4$ & $2,0 \pm 0,3$ \\
\hline PulsoO $_{2 \max }(\mathrm{ml} / \mathrm{bat})$ & $10,6 \pm 2,2$ & $11,1 \pm 2,0^{*}$ & $10,1 \pm 1,8$ & $10,1 \pm 1,5$ \\
\hline $\operatorname{LV}(\mathrm{ml} / \mathrm{kg} / \mathrm{min})$ & $19,1 \pm 5,0$ & $20,7 \pm 5,2$ & $18,1 \pm 3,7$ & $18,7 \pm 4,1$ \\
\hline FCLV (bpm) & $138,8 \pm 17,3$ & $144,0 \pm 15,8$ & $138,0 \pm 11,9$ & $138,6 \pm 16,5$ \\
\hline LV (I/min) & $1,1 \pm 0,4$ & $1,2 \pm 0,3$ & $1,0 \pm 0,2$ & $1,1 \pm 0,3$ \\
\hline PulsoO $_{2 \mathrm{LV}}(\mathrm{ml} /$ bat $)$ & $8,1 \pm 1,9$ & $8,4 \pm 1,8$ & $7,5 \pm 1,3$ & $7,7 \pm 1,2$ \\
\hline
\end{tabular}

Valores são média \pm desvio-padrão. $V^{2} \mathrm{O}_{\max }=$ Consumo máximo de oxigênio; $\mathrm{FCM}=$ Freqüência cardíaca máxima; $\mathrm{PulsoO}_{2 \max }=$ Pulso máximo de oxigênio; $\mathrm{VO}_{2 \mathrm{LV}}=$ Consumo de oxigênio do limiar ventilatório; FCLV = Freqüência cardíaca do limiar ventilatório; $\mathrm{PulsoO}_{2 \mathrm{LV}}=$ Pulso de oxigênio do limiar ventilatório. Para a comparação das variáveis antes e após 12 semanas foi utilizado o teste $t$ de Student para amostras pareadas para: $\mathrm{VO}_{2 \max }(\mathrm{ml} / \mathrm{kg} / \mathrm{min})(\mathrm{GC}), \mathrm{FCM}$ (GE e GC), $\mathrm{VO}_{2 \max }(\mathrm{l} / \mathrm{min})$ (GE e GC), Pulso $\mathrm{O}_{2 \max }\left(\mathrm{GE} \mathrm{e} \mathrm{GC),} \mathrm{FCLV} \mathrm{(GE} \mathrm{e} \mathrm{GC)} \mathrm{e} \mathrm{Pulso} \mathrm{O}_{2 L V}(\mathrm{GE})\right.$; para as variáveis: $\mathrm{VO}_{2 \max }(\mathrm{ml} /$ $\mathrm{kg} / \mathrm{min}$ ) (GE), LV (ml/kg/min) (GE e GC), LV (I/min) (GE e GC) e Pulso O O de Wilcoxon. * Indica diferença significante antes e depois do treinamento $(p<0,05)$. ${ }^{*}$ Indica diferença significante antes e depois do treinamento $(p<0,01)$. 


\section{DISCUSSÃO}

O consumo máximo de oxigênio e o limiar anaeróbio são considerados importantes indicadores da capacidade cardiorrespiratória. Neste estudo, o $\mathrm{VO}_{2 \max }$ aumentou em resposta ao treinamento de força no GE, sugerindo que este protocolo de treinamento de resistência de força é benéfico à potência aeróbia máxima. Entretanto, o limiar anaeróbio, determinado pelo método ventilatório e que é um importante indicador da capacidade aeróbia submáxima, se manteve inalterado depois de 12 semanas de treinamento de força no GE.

O aumento do $\mathrm{VO}_{2 \max }$ pode ser explicado devido ao protocolo de treinamento realizado, com alto número de repetições e curto intervalo entre as séries, sendo descrito como um treinamento de resistência de força com ênfase no metabolismo aeróbio, podendo acarretar alterações que proporcionam maior capacidade de captação de oxigênio pelos músculos ${ }^{(3,5)}$.

Esse aumento em resposta ao treinamento de resistência de força não é um resultado consensual, não sendo observado em alguns estudos $^{(12,13,19,24)}$, enquanto outros relataram aumento nesse índice ${ }^{(14,16)}$. Além disso, outra diferença do presente estudo se deve à participação exclusiva de mulheres, podendo sugerir o comportamento de determinado gênero em resposta ao treinamento; as voluntárias realizaram os testes cardiopulmonares antes e após 12 semanas, independente da fase do ciclo menstrual, pois, embora o controle dessa variável seja uma preocupação notória em estudos com mulheres, pesquisas anteriores demonstraram que a fase do ciclo menstrual não altera a capacidade cardiorrespiratória em mulheres ${ }^{(25,26)}$.

O fato de o limiar ventilatório não ter-se modificado pode ser explicado pela realização de treinamento com pesos e aparelhos de musculação, não sendo específico à corrida ou caminhada, já que os testes cardiopulmonares foram feitos em esteira rolante, ou seja, ergômetro não específico ao treinamento realizado, no qual os efeitos no limiar anaeróbio são menos evidenciados ${ }^{(8)}$. Além disso, o limiar anaeróbio pode ser influenciado pelo treinamento. diferentemente do $\mathrm{VO}_{2 \max }{ }^{(5)}$, já que o intervalo de oito a 12 semanas é o prazo indicado para a verificação das adaptações frente ao treinamento de força. Os resultados sugerem a necessidade de treinamento aeróbio, como caminhada ou corrida, para melhora do limiar anaeróbio em mulheres jovens não treinadas.

Santa-Clara et al.(12) avaliaram o efeito no consumo máximo de oxigênio e limiar ventilatório de um programa de um ano combinando treinamento resistido e aeróbio. Eles estudaram 40 homens com doença coronariana que foram divididos em três grupos: treinamento resistido mais aeróbio (grupo de exercício combinado), treinamento somente aeróbio e grupo controle. O grupo combinado aumentou o limiar ventilatório mais do que o grupo que treinou somente aeróbio, mas não aumentou o consumo máximo de oxigênio mais do que o grupo aeróbio. O presente estudo não apresentou melhora no limiar ventilatório, o que era esperado em resposta ao treinamento resistido, embora tanto os sujeitos quanto o protocolo de treinamento utilizado tenham sido diferentes da pesquisa acima.

Chtara et al. ${ }^{(16)}$ investigaram os efeitos do treinamento de força em homens durante 12 semanas, divididas em quatro períodos de três semanas, utilizando exercícios que focavam particularmente cadeias musculares envolvidas na corrida. O uso de exercícios de força intermitentes com curto intervalo de descanso mostrou afetar a capacidade cardiorrespiratória, porque aumentou o $\mathrm{VO}_{2 \max }$ e o segundo limiar ventilatório. Apesar de as características do treinamento de força desse estudo também serem diferentes das do presente trabalho, há concordância de que o treinamento de força possa aumentar o consumo máximo de oxigênio.

Poucos estudos examinaram o impacto do treinamento de resistência de força na capacidade aeróbia em mulheres. Bishop et al. ${ }^{(10)}$ não encontraram alterações no $\mathrm{VO}_{2 \max }$ e limiar de lactato depois do treinamento resistido em mulheres ciclistas treinadas em endurance e Hoff et al.(11) não encontraram mudanças no $\mathrm{VO}_{2 \max }$ e limiar de lactato em resposta ao treinamento de força máxima em mulheres atletas de cross-country. Entretanto, ambas as pesquisas estudaram os efeitos de protocolos de treinamento de força diferentes dos do presente estudo; determinaram o limiar de lactato e não o limiar ventilatório e avaliaram mulheres atletas, ou seja, com nível inicial de condicionamento físico muito maior do que o das voluntárias desta pesquisa, ficando muito mais difícil conseguir melhoras nas variáveis cardiorrespiratórias ${ }^{(18)}$.

O pulso de oxigênio depende do volume de $\mathrm{O}_{2}$ extraído dos tecidos periféricos e do volume captado pela circulação pulmonar durante cada batimento cardíaco; é igual à razão entre o consumo de oxigênio e a freqüência cardíaca para a mesma intensidade de esforço ${ }^{(8,9)}$. No pulso máximo de oxigênio houve aumento significativo para o GE quando comparado com o GC, sem diferença significativa na freqüência cardíaca máxima; isso sugere que o treinamento de resistência de força aumentou o volume de ejeção e/ou a captação periférica de oxigênio no exercício máximo.

Nenhuma alteração significativa no pulso de oxigênio do limiar anaeróbio foi observada, indicando que o protocolo de treinamento de resistência de força não proporcionou estímulo suficiente ao aumento do volume de ejeção ou captação periférica de oxigênio no limiar ventilatório.

Acreditamos que outros estudos são necessários para investigar os efeitos do treinamento de força na capacidade cardiorrespiratória de mulheres, usando diferentes protocolos de treinamento de força.

No grupo experimental foram observadas diferenças significantes na composição corporal. A análise das variáveis antropométricas mostrou aumento significante na massa magra, além de diminuição significativa no percentual de gordura e gordura corporal no GE. A composição corporal por dobras cutâneas é determinada por um método duplamente indireto, de modo que os valores de percentual de gordura, gordura corporal e massa magra são estimados. Sendo assim, o aumento na massa magra encontrado sugere hipertrofia muscular, adaptação induzida pelo treinamento, sendo esta hipótese reforçada pelo resultado do percentual de gordura, que mostrou importante redução, mesmo sem mudança significante na gordura corporal. Entretanto, não se pode afirmar que houve hipertrofia de fato, já que os valores da composição corporal são estimados. As mudanças encontradas na composição corporal neste estudo corroboram outros estudos que também relataram mudanças na composição corporal, pela avaliação das dobras cutâneas, em resposta ao treinamento de resistência de força em mulheres ${ }^{(27-29)}$.

A força muscular do GE aumentou, comprovada pelos testes de 1RM que apresentaram diferenças significativas em todos os exercícios na comparação com o GC. Esses resultados sugerem que o protocolo de treinamento com exercícios resistidos foi efetivo no aumento da força muscular. A melhora da força muscular em mulheres em resposta ao treinamento de força era esperada; os aumentos encontrados neste trabalho são similares aos de outros estudos com mulheres ${ }^{(28-30)}$. 
Os resultados encontrados neste estudo indicam que o protocolo de treinamento de força realizado proporcionou significante aumento da força muscular e da potência aeróbia das voluntárias, podendo ser adotado em protocolos de treinamento de mulheres jovens, trazendo importantes benefícios. Entretanto, para ocorrerem maiores adaptações cardiorrespiratórias, com melhora do limiar anaeróbio, é necessário a realização do treinamento aeróbio.

Assim, conclui-se que o presente protocolo de treinamento de resistência de força com alto número de repetições em mulheres jovens proporcionou melhora da potência aeróbia, evidenciada pelo aumento no $\mathrm{VO}_{2 \text { max }}$ e aumentou a força muscular, demonstrada pelos testes de 1RM, embora não tenha alterado o limiar ventilatório.

\section{AGRADECIMENTOS}

Os autores agradecem à Fundação de Amparo à Pesquisa do Estado de São Paulo (Fapesp) pelo suporte financeiro e bolsas de treinamento técnico, à Coordenação de Aperfeiçoamento de Pessoal de Nível Superior (Capes) pela bolsa de mestrado e ao Conselho Nacional de Desenvolvimento Científico e Tecnológico (CNPq) pela bolsa de iniciação científica.

$\overline{\text { Todos os autores declararam não haver qualquer potencial conflito de }}$ interesses referente a este artigo.

\section{REFERÊNCIAS BIBLIOGRÁFICAS}

1. McCartney N, Moroz D, Garner SH, McComas AJ. The effects of strength training in patients with selected neuromuscular disorders. Med Sci Sports Exerc 1988;20:362-8.

2. McCartney N, McKelvie RS. The role of resistance training in patients with cardiac diseases. J Cardiovasc Risk 1996;160-6.

3. Kraemer WJ, Ratamess NA. Fundamentals of resistance training: progression and exercise prescription. Med Sci Sports Exerc 2004;36:674-88.

4. Gobbi S, Villar R, Zago AS. Bases teórico-práticas do condicionamento físico. Rio de Janeiro: Editora Guanabara Koogan, 2005.

5. American College of Sports Medicine. The Recommended Quantity and Quality of Exercise for Developing and Maintaining Cardiorespiratory and Muscular Fitness and Flexibility in Healthy Adults. Med Sci Sports Exerc 1998;30:975-91.

6. Poole DC, Gaesser GA. Response of ventilatory and lactate thresholds to continuous and interval training. J Appl Physiol 1985;58:1115-21.

7. Cesar MC, Pardini DP, Barros Neto TL. Efeitos do exercício de longa duração no ciclo menstrual, densidade óssea e potência aeróbia de corredoras. Revista Brasileira de Ciência \& Movimento 2001;9:7-13.

8. Barros Neto TL, Cesar MC, Tambeiro VL. Avaliação da aptidão física cardiorrespiratória In: Ghorayeb N, Barros Neto TL. O exercício: preparação fisiológica, avaliação médica, aspectos especiais e preventivos. São Paulo: Editora Atheneu, 1999;3-13.

9. Wasserman K, Hansen JE, Sue DY, Casaburi R, Whipp BJ. Principles of exercise testing and interpretation. 3rd ed. Baltimore: Lippincott Williams \& Wilkins, 1999.

10. Bishop D, Jenkins DG, Mackinnon LT, McEniery M, Carey MF. The effects of strength training on endurance performance and muscle characteristics. Med Sci Sports Exerc 1999;31:886-91.

11. Hoff J, Helgerud J, Wisloff U. Maximal strength training improves work economy in trained female cross-country skiers. Med Sci Sports Exerc 1999;31:870-7.

12. Santa-Clara H, Fernhall BO, Mendes M, Sardinha LB. Effect of a year combined aerobic and weighttraining exercise programme on aerobic capacity and ventilatory threshold in patients suffering from coronary artery disease. Eur J App Physiol 2002;87:568-75.

13. Ferrara CM, McCrone SH, Brendle D, Ryan AS, Goldeberg AP. Metabolic effects of the addition of resistive to aerobic exercise in older men. Int J Sport Nutr Exerc Metab 2004;14:73-80.

14. McCarthy JP, Agre JC, Graf BK, Pozniak MA, Vailas AC. Compatibility of adaptive responses with combining strength and endurance training. Med Sci Sports Exerc 1995;27:429-36.

15. Antoniazzi RMC, Portela LOC, Dias JFS, Sá CA, Matheus SC, Roth MA, et al.. Alteração do $V_{2}{ }_{2 m a x}$ de indivíduos com idades entre 50 e 70 anos, decorrente de um programa de treinamento com pesos. Atividade Física \& Saúde 1999;4:27-34.

16. Chtara M, Chamari K, Chaouachi M, Chaouachi A, Koubaa D, Feki Y, et al.. Effects of intra-session concurrent endurance and strength training sequence on aerobic performance and capacity. $\mathrm{Br} J$ Sports Med 2005;39:555-60.

17. Tanaka $H$, Swensen $T$. Impact of training on endurance performance. A new form of cross-training? Sports Med 1998;25:191-200

18. Wilmore JH, Costill DL. Fisiologia do esporte e do exercício. 2a. ed. São Paulo: Editora Manole, 2001.

19. Glowacki SP, Martin SE, Maurer A, Baek W, Green JS, Crouse SF. Effects of resistance, endurance and concurrent exercise outcomes in men. Med Sci Sports Exerc 2004;36:2119-27.

20. Guedes DP, Guedes JERP. Controle do peso corporal: composição corporal, atividade física e nutrição. Londrina: Ed. Midiograf, 2003.

21. Furtado $E$, Simão $R$, Lemos $A$. Análise do consumo de oxigênio, freqüência cardíaca e dispêndio energético, durante as aulas do jump fit. Rev Bras Med Esporte 2004;10:371-5.

22. Brown LE, Weir JP. (ASEP) Procedures recommendation I: accurate assessment of muscular strength and power. Journal of Exercise Physiology 2001;4:1-21.

23. Zar JH. Biostatical analysis. 3rd ed. New Jersey: Prentice Hall, 1999

24. Cauza E, Hanusch-Enserer U, Strasser B, Ludvik B, Metz-Schimmerl S, Pacini G, et al. The relative benefits of endurance and strength training on the metabolic factors and muscle function of people with type 2 diabetes mellitus. Arch Phys Med Rehabil 2005;86:1527-33.

25. De Souza MJ, Maguire MS, Rubin KR, Maresh CM. Effects of menstrual phase and amenorrhea on exercise performance in runners. Med Sci Sports Exerc 1990;22:575-80.

26. Dean TM, Perreault L, Mazzeo RS, Horton TJ. No effect of menstrual cycle phase on lactate threshold. J Appl Physiol 2003;95:2537-43.

27. Roth SM, Martel GF, Ivey FM, Lemmer JT, Metter EJ, Hurley BF, et al. High volume, heavy-resistance strength training and muscle damage in young and older women. J Appl Physiol 2000;88:1112-8.

28. Hurlbut DE, Lott ME, Ryan AS, Ferrell RE, Roth SM, Ivey FM, et al. Does age, sex or ACE genotype affect glucose and insulin responses to strength training? J Appl Physiol 2002;92:643-50.

29. Poehlman ET, Denino WF, Beckett T, Kinaman KA, Dionne IJ, Dvorak R, et al. Effects of endurance and resistance training on total daily energy expenditure in young women: a controlled randomized trial. J Clin Endocrinol Metab 2002;87:1004-9.

30. Dias RMR, Cyrino ES, Salvador EP, Nakamura FY, Pina FLC, Oliveira AR. Impacto de oito semanas de treinamento com pesos sobre a força muscular de homens e mulheres. Rev Bras Med Esporte 2005;1 1:224-8. 